\title{
Category: Epistemology
}

\section{A Short Refutation of Strict Normative Evidentialism}

Abstract: This paper shows that strict evidentialism about normative reasons for belief is inconsistent with taking truth to be the source of normative reasons for belief. It does so by showing that there are circumstances in which one can know what truth requires one to believe, yet still lack evidence for the contents of that belief.

\section{Introduction}

Strict normative evidentialism is the view that all normative reasons for belief are evidential reasons. ${ }^{1}$ In this paper, I show that the falsity of strict normative evidentialism-just 'evidentialism' from here forward-follows from weak and uncontroversial premises. I argue that none of the premises may be plausibly rejected, and thus that evidentialism is false. ${ }^{2}$

\section{Unstable beliefs with many fixed points}

Evidentialism fails straightforwardly, at least on common views about evidence, for certain classes of truths. Reasons to believe logical truths, mathematical truths, or certain $a$ priori truths are generally non-evidential. However, it is often touted as the right view for

\footnotetext{
${ }^{1}$ Strict evidentialism is a widely held view. See Adler (2002), Hieronymi (2005), Kelly (2002), Parfit (2001), Shah (2003 \& 2006), Skorupski (2011), Wedgwood (2002), and Yamada (2010) for examples.

${ }^{2}$ For an in depth discussion of other aspects of the many fixed points numbers game, see [AUTHOR].
} 
empirical truths. For at least one class of empirical truths, however, it can also fail. A thought experiment illustrates the category.

Alice is the subject of a psychology experiment. She knows all of its rules and parameters. The experiment goes as follows: Alice is attached to a computer that can read her beliefs accurately and reliably. In front of her is a screen that is connected to the computer. Alice is asked to predict what number will appear on the screen. The rules (of which Alice is fully aware) for generating this number are as follows:

The computer will scan for Alice's belief about what number will appear on the screen, and then after a one minute delay, will put up a number on the screen based on Alice's belief about what number will appear on the screen. The computer determines what number will appear on the screen in the following way: Let $n$ be the number that Alice believes will appear on the screen. If $n \geq 0$, then the number that will appear on the screen is $1 / 2 n+1$. If $n<0$, then the number that will appear on the screen is $1 / 2 n-1$. If Alice changes her belief during the delay, the computer will take $1 / 2 n+1$ or $1 / 2 n-1$, as appropriate, of the new number and display it instead one minute from the change of belief. $^{3}$

If Alice suspends judgement or has no view about what number will appear on the screen, has multiple beliefs about what number will appear on the screen, believes that

\footnotetext{
${ }^{3}$ This procedure applies only in the case where Alice has a single belief about what number will appear on the screen, and her belief concerns a specific, single number (i.e. not a disjunctive belief or a belief just that some unidentified number will appear).
} 
multiple numbers will appear on the screen, has a disjunctive belief about what number will appear on the screen, or has a belief that is a category error (e.g. that the number 'Herbert' will appear on the screen), then the number 16 will appear on the screen.

Two final remarks should be made about Alice. She has no particular dispositions concerning what to believe about what number will appear on the screen, nor does she have any beliefs about her dispositions to do so. And, Alice's relevant mental states are luminous to her. The former point is of special importance, given the discussion of evidence below. If Alice had, or believed that she had, a disposition to believe that the number on the screen will be 2 , then she would have evidence that the number on the screen will be 2 (given that her belief would cause that result).

Given these parameters, Alice can see that there are only two fixed points and hence only two stable belief states: that the number on the screen will be $2(1 / 2 \cdot 2+1=2)$, or that the number on the screen will be $-2(1 / 2 \cdot-2-1=-2)$. Suspension of judgement or failure to form a belief about what number will appear in the screen is not stable, because Alice will know that, having suspended judgement, the number on the screen will be 16. Assuming for the sake of argument that she will make the relevant inference (and knows that she will do so), then Alice will infer from her having suspended judgement that the number on the screen will be 16 , thereby leading her to form the belief that the number on the screen will be 16 . Having formed that belief, she knows that the computer will rescan, and the number will be 9 $(1 / 2 \cdot 16+1=9)$, and then this pattern will repeat indefinitely. 


\section{Fixed points and permissibility}

Intuitively, it is permissible for Alice to believe that the number that will appear on the screen will be 2 , and it is permissible for Alice to believe that the number on the screen will be -2. To be precise:

Permissible (believe 2 or believe -2) \& Permissible (believe 2 and -2).

Intuitively, too, it is impermissible for Alice to believe that the number that will appear on the screen is anything else, and it is impermissible for Alice to suspend judgement or not have a belief. To be precise:

Let $y$ be that a particular number (or multiple numbers, or other content), other than 2 or -2 , will appear on the screen.

and

Not permissible (believe $y$ or suspend judgement).

We should accept the following inference pattern:

1. Necessarily $(a \leftrightarrow b)$

2. Permissible $a$ 


\section{$\therefore$ 3. Permissible $b^{4}$}

Applying this inference pattern, we can generate a new argument for an ought rather than a weaker permission:

Let $p$ be that you believe that the number on the screen will be 2 and only have that belief about the number on the screen, or you believe that the number on the screen will be -2 and only have that belief about the number on the screen.

Let $q$ be that you suspend judgement or you believe that the number that will appear on the screen will be something other than 2 or -2 , and only have that particular belief about what will appear on the screen.

$$
\begin{aligned}
& \text { 1. Necessarily }(\neg p \leftrightarrow q) \\
& \text { 2. } \neg \text { Permissible }(q) \\
& \therefore 3 . \neg \text { Permissible } \neg p^{5}
\end{aligned}
$$

Not permissible not $p$ is the equivalent of ought $p$. Oughts are stronger than reasons, and ought $p$ implies normative reasons $p$. If one (epistemically) ought to believe $b$, then one has a

\footnotetext{
${ }^{4}$ This inference pattern would be a semantic consequence of a deontic logic that treated propositions as sets of possible worlds, and it is consistent with other standard accounts of propositions. It also holds for any variant of standard deontic logic. It remains plausible independently of whether or not one endorses SDL. Importantly, the inference in the case of necessary coextension does not fall prey to the counterexamples that undermine the undirectional cousin of this inference pattern, sometimes called deontic inheritance.

${ }^{5}$ I am assuming the same inference pattern as above holds with the addition of a 'not' operator.
} 
normative epistemic reason to believe $b$. The reverse, of course, is not the case. So, even on the weak assumption that one is permitted to believe that either of the fixed points will appear on the screen and is not permitted to suspend judgement or to have other beliefs about what number will appear on the screen, there is an epistemic reason to believe one of the fixed points.

\section{Explaining the permission}

In the previous section, I observed that it was intuitively that it was permissible to believe either of the fixed points in the numbers game experiment. A better explanation can be offered. We should accept a principle, negative knowledge:

Negative Knowledge: If you know that $p$ is false, then you are not permitted to believe $p$.

And we should also accept non-suspension:

Non-suspension: If you know that $p$ is the case, then you are not permitted to suspend judgment towards $p$.

Negative knowledge follows from the factivity of knowledge and the more general view that epistemic normative reasons for belief are truth directed. Non-suspension follows from the same. Negative knowledge rules out believing anything other than one of the fixed points. Non-suspension rules out suspending judgement, because one knows that suspension of 
judgement will result in the number 16 appearing on the screen. Believing the fixed points is not ruled out by either of these principles, nor by negative evidence (that one will not believe that the number on the screen will be 2 or that it will be -2). So, it is permissible to believe either of the fixed points.

At the same time, there is no evidence, given the stipulations of the thought experiment, that Alice will believe that the number on the screen will be 2 in particular, or that she will believe that number on the screen will be -2 in particular. Since it is Alice's actual belief that determines which number appears on the screen, only evidence concerning what Alice will believe can serve as evidence for what number will appear on the screen. Thus, the permissibility does not derive from evidential considerations.

\section{Conclusion}

I have shown in this paper that the falsity of strict normative evidentialism, even for beliefs concerning empirical truths, follows from a set of uncontroversial premises. This conclusion is consistent with the weaker view, that in cases to which evidential reasons apply, they are the only applicable reasons. At best, then, slack normative evidentialism may be a correct view about normative reasons for belief, but evidence cannot do all the work. 


\section{Works Cited}

Adler, Jonathan (2002). Belief's Own Ethics. New York: Bradford Books.

Hieronymi, Pamela. (2005). The wrong kind of reason. The Journal of Philosophy, 102: 437-457.

Kelly, Thomas. (2002). The rationality of belief and some other propositional attitudes.

Journal of Philosophical Research, 110, 163-196.

Parfit, Derek. (2001) Reasons and rationality. In D. Egonsson, J. Josefsson, B. Petersson, and

T. Rønnow-Rasmussen (Eds.) Exploring Practical Rationality (pp. 17-39). Aldershot:

Ashgate.

Shah, Nishi (2003). How truth governs belief. Philosophical Review 112 (4):447-482.

Shah, Nishi (2006). A new argument for evidentialism. Philosophical Quarterly 56 (225):

481-498.

Skorupski (2011). The Domain of Reasons. Oxford: Oxford University Press.

Wedgwood, Ralph (2002). The aim of belief. Philosophical Perspectives 16:267-97.

Yamada, Masahiro (2010). A new argument for evidentialism? Philosophia 38 (2):399-404. 